\title{
Short-term prediction of the power generation of wind turbines
}

\author{
Á. Székely, T. Barbarics \\ Group of Electromagnetic Theory, Dept. of Broadband Infocommunication and Electromagnetic Theory, \\ Faculty of Electrical Engineering, Budapest University of Technology and Economics \\ 1111 Egry József u. 18. Budapest, Hungary \\ Phone: +361 4633190, Fax:+361 4633189, e-mail: szekely@evtsz.bme.hu ; barbarics@evtsz.bme.hu
}

\begin{abstract}
Since the erection of the first wind turbine in Hungary in 2001, the proportion of power generated from wind has increased considerably, furthermore an even faster growth is expected in the next years. However, the more widespread use of wind energy plays a role in the fact that deviations from the international exchange schedule are becoming more frequent.

In this paper we summarize the present situation concerning the usage of wind power in Hungary, detail the results and the limitations of our investigations carried out on the public data of the meteorological forecasts that are the primary sources of power generation schedules of wind power plants and suggest possible improvements on the reliability of the schedules based on the forecasts.
\end{abstract}

\section{Key words}

Wind power, meteorological forecast, power generation schedules, simulation

\section{Introduction - Usage of wind power in Hungary}

The energy of wind has been utilized already in the first decades of the 20th century in Hungary by wind mills. This means that in contrast with some opinions, there exist certain areas of the country where exploitation of wind energy can be rewarding. We can easily obtain information about the location of these areas by simply looking at the map of former wind mills which is shown on Fig. 1. [1] These early turbines were used to intermittently operate or aid the operation of devices, mostly water pumps. The turbines were isolated and there was no need to consider the strict technical criteria that working as a unit of the modern power system requires.

As of January, 2006 there are altogether 7 wind turbines operating in Hungary, capacity of which is between 0,5 and $1 \mathrm{MW}$ each. The total built-in capacity of the power plants in Hungary is $8831 \mathrm{MW}$ (as of 31 December, 2004), 21 per cent of which is the nuclear power plant at Paks. The weekly maximal system load varies between

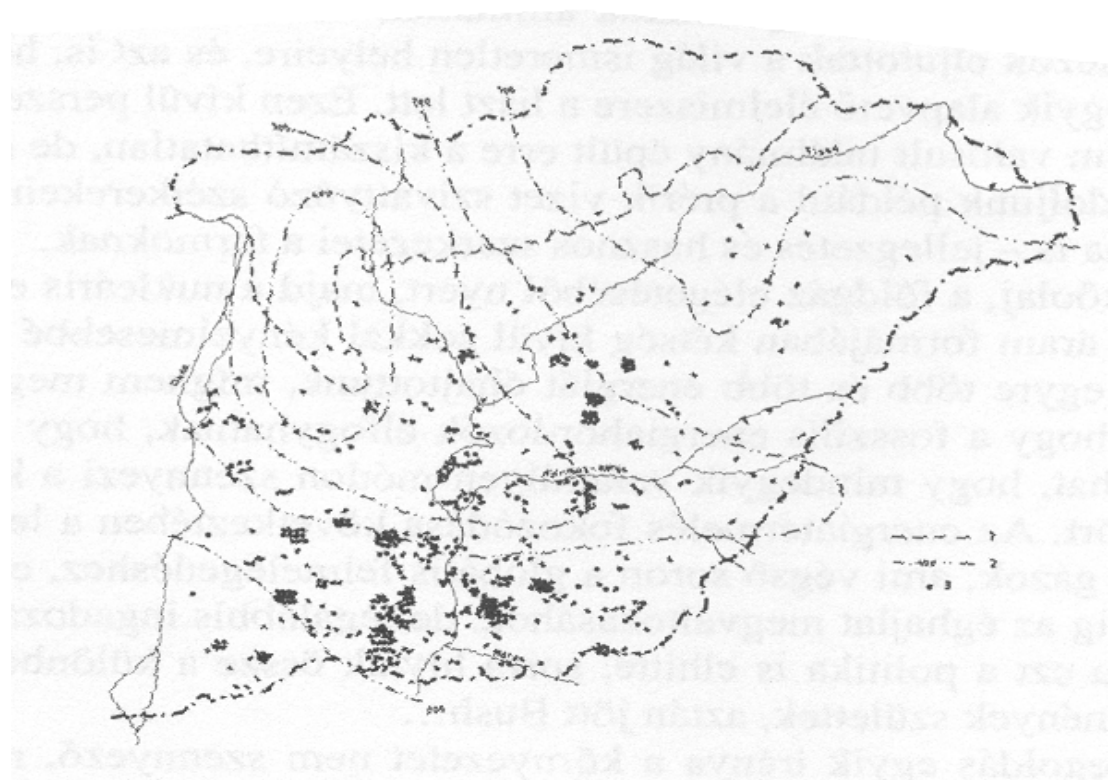

Fig. 1. Location of wind mills in Eastern Hungary in the 1910s 
4500 and $6000 \mathrm{MW}$. [2] As it can be seen from the above data, the present effect of the wind power plants on the Hungarian power system is practically negligible. In some cases, investors contract with villages that use the generated electricity for e.g. street lightning and sell the excess power to the local power supplier company.

However, the new Law of Electric Power prescribes obligatory acceptance and a price considerably higher than average for the power generated using renewable energy sources. Therefore, there are several major wind farms that are in the stage of planning and collecting permissions. The Hungarian Energy Office is making decisions this year on new wind power facilities with a total built-in power of over $150 \mathrm{MW}$, and a further 1000 MW is under planning by various investment groups.

According to the director of the Hungarian Power System Operator Company (MAVIR), the power grid in its present state is capable of regulating the voltage within the prescribed range and neutralize the deviations from the schedule caused by wind turbines as long as the total builtin capacity of these does not exceed 150 MW. [3]

Apart from causing fluctuations in voltage, compulsory acceptance of wind power often makes necessary to regulate the system by decreasing the generation of much cheaper basic power plants, in some cases even the nuclear plant. These measures cause not only excess costs concerning the whole system but also decrease the lifespan of machine units of the base power plants.

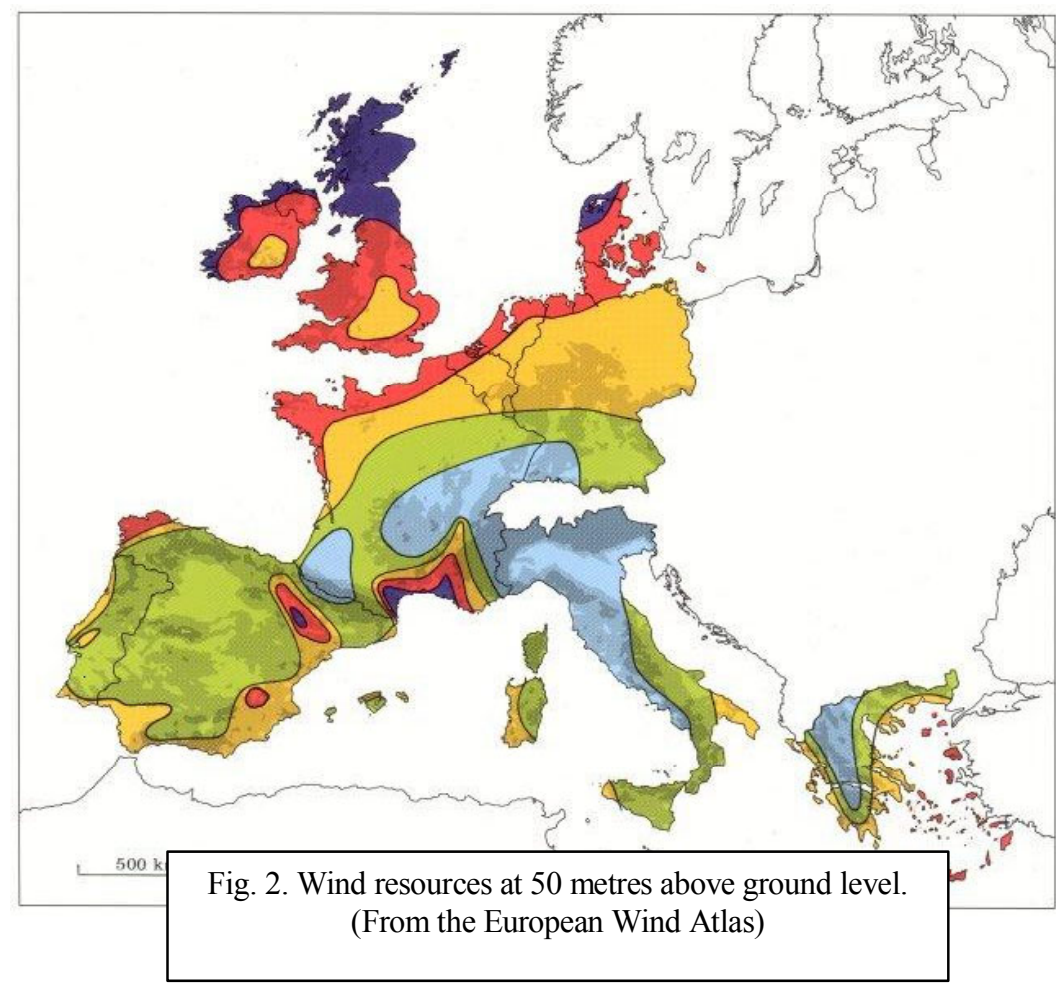

Presently, companies operating a wind turbine are obliged to give a daily planned schedule for the amount of generated power but due to the obligatory acceptance, they are not responsible for the accuracy of data or deviations from the schedule. Presently, this does not cause major problems, but effect of wind turbines will increase very rapidly in the next 5 years.

Due to the reasons mentioned, it is necessary to know basic facts about the types, reliability and limitations of available meteorological wind forecasts. We have collected a sample of data of the public meteorological forecasts in Hungary and carried out statistical investigations to determine their accuracy. In the last section we summarize several possibilities for improving the reliability of power schedules.

\section{Wind forecasts}

\section{A. Long-term , wind-maps”}

So-called wind maps are based on a large amount of data collected from several meteorological measurement stations that are processed to produce a continuous map with the average wind speeds for a larger area. The most widespread such map is the European Wind Atlas that was constructed by the Risø National Laboratories in Roskilde, Denmark. [4]

Figure 2 shows the overview map of Western Europe with annual average wind speeds (black and red colors show the highest averages above $6 \mathrm{~m} / \mathrm{s}$ ). In the Wind Atlas, more detailed map sections are also available for the countries of Western Europe. 


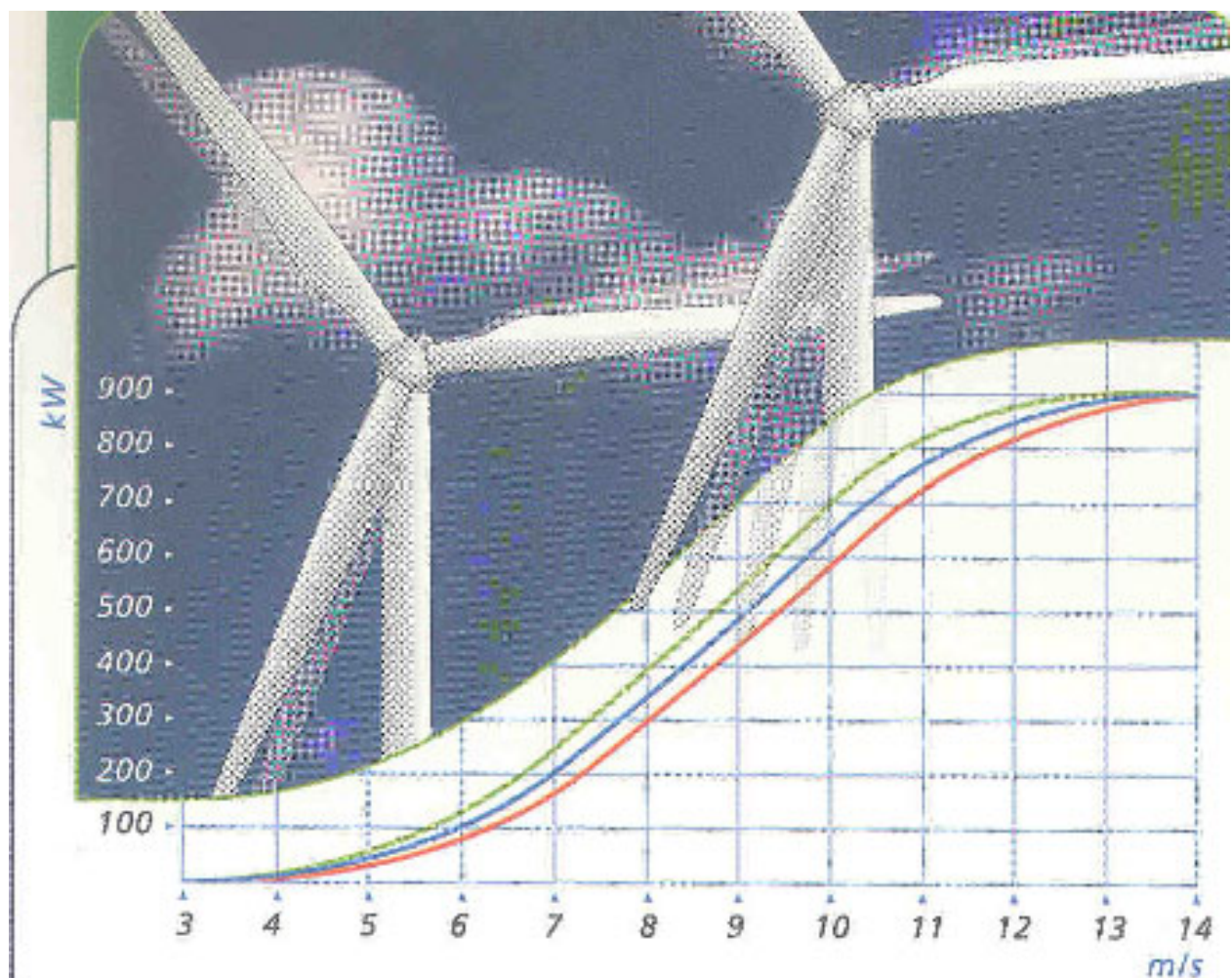

Enros Wind 900 si

Enron Wind 900 s

Fig. 3. Generated power vs. wind speed characteristic of the Enron Wind 900S type wind turbines

rotors move is proportional to the third power of the speed of wind, therefore finding a site with 10 per cent higher annual average wind speed can result in about 30 per cent increase of the amount of produced power.

However, these models cannot be used for providing short-term schedules. One reason for this lies in the statistical nature of calculations involved that makes the method optimal only for calculating long-term averages. Another reason is that the amount of calculations is far too high for these models to be applied as a daily routine.

\section{B. Short-term meterorological wind forecasts}

Meteorological forecasts are based on numerical solutions of the extremely complex differential equation systems that describe the processes of the atmosphere. The accuracy and spatial resolution of these numerical solutions are largely determined by the accuracy of boundary conditions and the available computational capacity.

Hungarian meteorological wind forecasts are today based on the MM5 system, however, our investigations were made using the output data from the previous, ALADIN system. The computational capacity has limited the spatial resolution to 6500 metres (as of June, 2004).

This resolution obviously makes it impossible to take into consideration the flowmodifying effects detailed in the previous point.

In order to give an estimation for the accuracy of meteorological forecasts, we have collected a sample of forecast and measurement data between 7 March and 5 April, 2004 every 6 hours from measurement stations in 7 major cities of Hungary. The forecasted and actual power generation data were calculated using the characteristic of the Enron Wind 900S turbine, which is shown on Figure 3.

From the sample of data, the correlation coefficients of forecasted and measured data have been calculated and evaluated. Figure 4 shows the results as a function of the correlation of the data series of forecasted and measured wind speeds vs. the timespan of forecasts (in days). The collected data shows that the correlation improves when the time-span of the forecast decreases, but it does not exceed the value of 0.7 . It is interesting to observe that the data collected from the station at Miskolc clearly stands out from other data series with a much lower forecast correlation to the measured data. The explanation for the phenomenon is that Miskolc is bordered from the west and north by mountaneous areas with complex terrain that make winds less predictable in the area.

We have to mention that on demand, more accurate forecasts with a finer spatial resolution are also available.

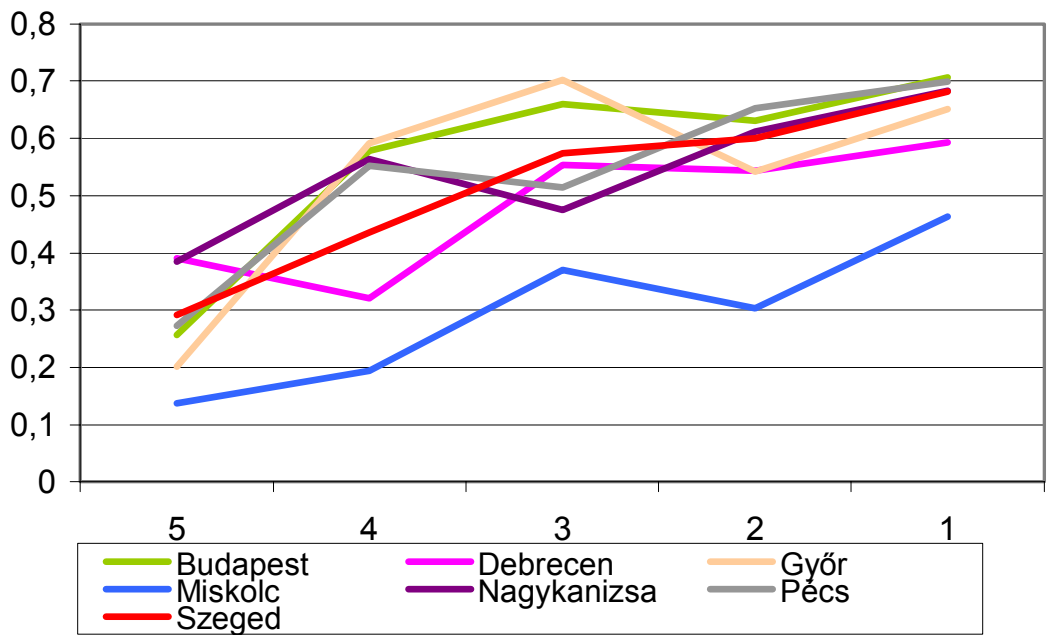




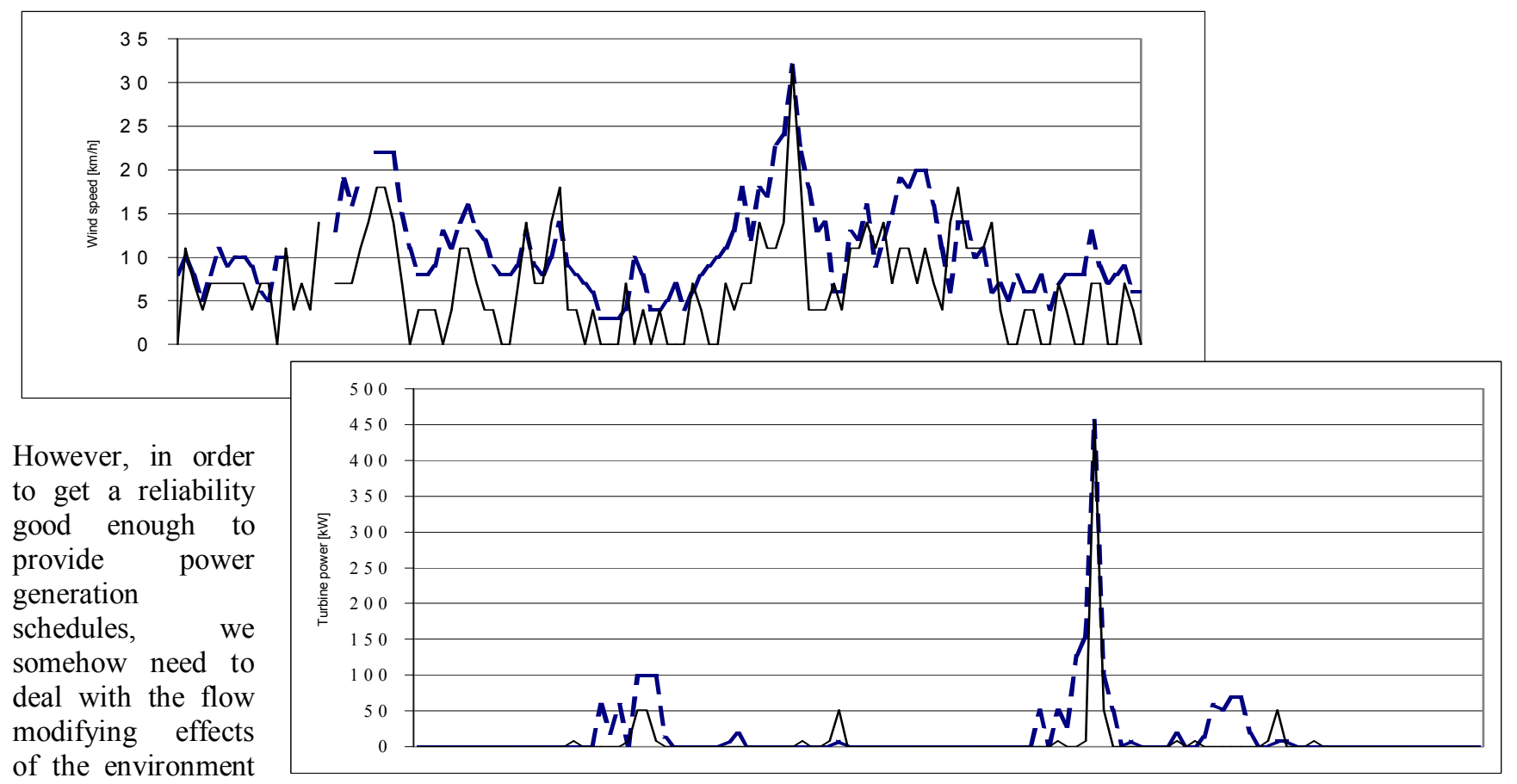

of the turbine. In the next section we examine some possibilities to do this improvement.

Fig. 5. Results. A typical data series of forecasted and measured wind speeds (above) and the calculated power to be generated .

\section{Possibilities of forecast improvement}

\section{A. Flow-dynamical modeling}

Apparently, the most trivial solution for taking into consideration the terrain and objects in the environment of the wind turbine could be measuring the wind speed at the location of the turbine on a scaled model, using a wind channel measurement system. Measuring several characteristic wind directions, a database could be constructed, containing the measurement data for the turbine site for several meteorological situations. The drawback of this solution is that calculating the correct scaling of the model, building it and measuring in several positions would imply such high costs that are not likely to return under present conditions.

Another possibility is computer-aided modeling of the terrain and determining expected wind speed at the site of the wind power plant, considered the forecasts for some surrounding points are known. The problem with this method is that output data from meteorological forecasting models cannot be directly used as an input data or boundary condition for the presently used flowdynamic computational softwares. A further difficulty is that only the input of a sufficiently detailed digital map of an area of 30 to $40 \mathrm{~km}^{2}$ would take about two months for a flow-dynamics expert.

In spite of these drawbacks, computer-aided flow modeling is used successfully e.g. in Germany, where specialized companies make the necessary orographic measurements, construct the digital map of the area and continuously provide the improved forecasts to the plant and the power system operator. We have to mention that the proportion of wind power in Germany is several times higher than that in Hungary, therefore these laborintensive methods can be more rewarding.

\section{B. Statistical approach}

Our suggestion for the problem is a phenomenological approach that does not deal with the reasons of the difference of wind speed between the point of forecast data and location of the turbine, instead tries to determine weighting coefficients for the surrounding 4 or 16 forecast points in a way that the calculated forecast data has a minimal RMS error compared to measured data on a sample data series.

Input data for this method would be the meteorological forecast for the surrounding points and wind speed measurement data from the wind turbine, that is available irrespectively from the usage of this method.

To determine the optimal weighting coefficients, statistical computations are necessary, complexity of which can raise too high in case of larger wind farms. Therefore in the next point we suggest a basicly different method for the above detailed task.

\section{Genetic algorythms in general}

Genetic algorythms (or evolutionary methods) is a branch of artificial intelligence research, first mentioned in the 1960 s, and include several methods the common basis of which is to generate a first so-called population of guesses for the optimal solution, that are chosen from a „search space", i.e. all possible solutions of a certain problem, then calculate the error of these solutions and choose the „,best” ones. From the chosen solutions we can generate a second population by randomly changing 
some of the bits and combining parts of the solutions with each other. From this second population the best data can again be chosen and the process can be repeated arbitrarily many times until a final solution is reached.

Parameters of the solution method are the number of guesses in a population, the probability and number of changes made on them and the maximal number of iterations or the limit of the error compared to the optimal solution that is once reached, the iteration can be stopped. These parameters can be set in order to get better solutions at every iteration, but their optimal value differs for all types of problems, and are usually determined rather empirically than following theoretical considerations. [6]

From the above short summary it is clear that the most important criteria for usability of genetic algorythms for solving a certain problem are:

- a particular solution of the problem can be easily encoded to a binary number

- $\quad$ the error of a particular solution compared to the optimal can be quantified

We can say that the problem of finding the optimal weighting coefficients for providing wind forecast for the location of the turbine perfectly fulfills the above criteria, therefore our Department is planning to continue the work together with engineering students to perform experimental computations in order to check the efficiency and quality of the detailed methods in practice for this type of problem.

\section{Conclusion}

The growing weight of wind power in Hungarian power industry makes more accurate wind speed predictions inevitably necessary, however, the limitations of meteorological forecasts do not allow much higher reliability than presently.

The methods suggested in this paper are relatively simple algorythms that can be also available for institutions with smaller computational capacity than meteorological institutes. The labor need and cost of the suggested methods is considerably smaller than that of flow- dynamical modeling that requires numerous on-site measurements and a large amount of work and time, therefore the method detailed above can be remunerative even for smaller wind farms that are currently being planned in Hungary. Further applications in other fields of industry are also possible.

We have to mention that the applicability and method is not yet tested for the certain problem, but we hope that we will be able to continue the work and carry out successful and efficacious trial computations.

\section{Acknowledgement}

The authors of the article wish to acknowledge the financial support for the participation on the conference that was provided by the Foundation for the Education of Energetic Engineers.

\section{References}

[1] Tar, Keveyné, Tóth, Vágvölgyi: Possibilities of utilizing wind energy in Hungary. Proc. of Conference of Meteorological Sciences, Budapest (Hungary), 2001

[2] Data of the power system of Hungary 2004. Hungarian Power System Operator Company

[3] Antal Tombor: Problems of the safety of electricity supply under market environment. Proc. of the 52nd Conference of the Hungarian Electrotechnic Association, Eger (Hungary), August 2005

[4] Homepage of Risø National Laboratories, Roskilde, Denmark: www.risoe.dk

[5] Kornélia Radics: Wind climate of Hungary, utilization of wind energy. Proc. of the 5th Conference of Pro Scientia Medalists, Sopron (Hungary), 2000

[6] Marek Obitko: Introduction to genetic algorythms. (Czech Technical University, Praha, Czech Rep.) cs.felk.cvut.cz/ xobitko/ga 